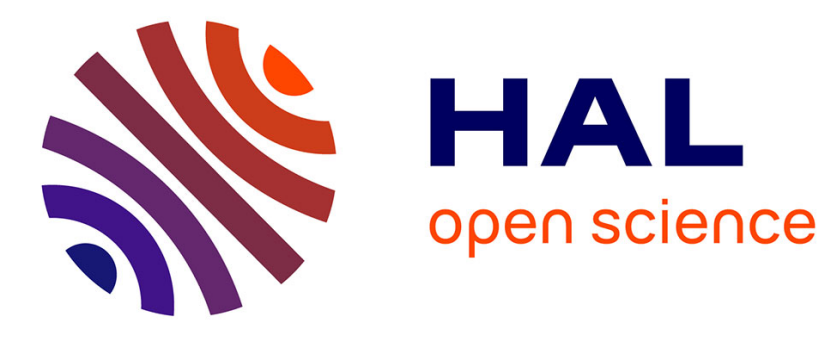

\title{
Self-Similar Relaxation of Confined Microfluidic Droplets
}

Margaux Kerdraon, Joshua D Mcgraw, Benjamin Dollet, Marie-Caroline

Jullien

\section{- To cite this version:}

Margaux Kerdraon, Joshua D Mcgraw, Benjamin Dollet, Marie-Caroline Jullien. Self-Similar Relaxation of Confined Microfluidic Droplets. Physical Review Letters, 2019, 123 (2), 10.1103/PhysRevLett.123.024501 . hal-02278038

\section{HAL Id: hal-02278038 https://hal-univ-rennes1.archives-ouvertes.fr/hal-02278038}

Submitted on 15 Jun 2020

HAL is a multi-disciplinary open access archive for the deposit and dissemination of scientific research documents, whether they are published or not. The documents may come from teaching and research institutions in France or abroad, or from public or private research centers.
L'archive ouverte pluridisciplinaire HAL, est destinée au dépôt et à la diffusion de documents scientifiques de niveau recherche, publiés ou non, émanant des établissements d'enseignement et de recherche français ou étrangers, des laboratoires publics ou privés. 
Version June 15, 2020

\title{
Self-similar relaxation of confined microfluidic droplets
}

\author{
Margaux Kerdraon, ${ }^{1}$ Joshua D. McGraw, ${ }^{1}$ Benjamin Dollet, ${ }^{2}$ and Marie-Caroline Jullien ${ }^{1,3}$ \\ ${ }^{1}$ Gulliver CNRS UMR 7083, PSL Research University, \\ ESPCI Paris, 10 rue Vauquelin, 75005 Paris, France \\ ${ }^{2}$ Univ. Grenoble Alpes, CNRS, LIPhy, 38000 Grenoble, France \\ ${ }^{3}$ Univ Rennes 1, CNRS, IPR (Institut de Physique \\ de Rennes) - UMR 6251, F-35000 Rennes, France
}

(Dated: June 15, 2020)

\begin{abstract}
We report an experimental study concerning the capillary relaxation of a confined liquid droplet in a microscopic channel with rectangular cross-section. The confinement leads to a droplet that is extended along the direction normal to the cross-section. These droplets, found in numerous microfluidic applications, are pinched into a peanut-like shape thanks to a localized, reversible deformation of the channel. Once the channel deformation is released, the droplet relaxes back to a plug-like shape. During this relaxation, the liquid contained in the central pocket drains towards the extremities of the droplet. Modeling such visco-capillary droplet relaxation requires considering the problem as 3D due to confinement. This 3D consideration yields a scaling model incorporating dominant dissipation within the droplet menisci. As such, the self-similar droplet dynamics is fully captured.
\end{abstract}


The relaxation dynamics of liquid interfaces towards equilibrium induced by surface tension has been studied for many decades [1 7]. The gradient of capillary pressure determined by the interface curvature along the deformed surface generates a flow, which in turn is limited by viscous stresses. In practical microfluidic systems, emulsions, foams or porous media, droplet interfaces are constantly deformed and relax [8-12], in particular owing to the complex topography of real systems [13, 14]. In the context of droplet relaxation, experimental, theoretical and numerical studies considered a droplet immersed in an infinite bath [2, 15, 16] or confined by two parallel walls [12, 17, 18]. As such, droplet relaxation could be reduced to one of its planes of invariance [2, 6, 12]. Such simplifications aid in the analytic resolution of dynamical relaxation problems. However, for microfluidic droplets confined in both width and height, those symmetries cannot be invoked. In this Letter we instead propose a model that accounts for the intrinsic 3D nature of the observed microfluidic flow.

We study the relaxation of a plug-like droplet forced out of equilibrium in a rectangular geometry, representing a large number of microfluidic applications [19]. Typical deformations are shown in Fig. 1. The interface has a pocket-like profile composed of a reservoir followed by what resembles a thin film in an equatorial observation plane. Critically though, a meniscus runs along the contour of the droplet, and gutters are formed at the four corners of the channel cross-section. Our analysis shows that these gutters play a key role in the observed relaxations. Despite the complexity of the 3D geometry of such droplets, we find that a scaling model balancing the capillary driving force with the viscous dissipation in the gutters and menisci allows us to collapse all experimental relaxation data.

In our experiments the outer liquid is a solution of sodium dodecyl sulfate (SDS, purchased from Sigma Aldrich) at concentration $2.94 \mathrm{~g} \cdot \mathrm{L}^{-1}$ in ultrapure water (MilliQ). The surfactant concentration is above the critical micellar concentration $\left(c_{\mathrm{cmc}}=1.92 \mathrm{~g} \cdot \mathrm{L}^{-1}\right)$ ensuring a constant surface tension, $\gamma=11 \pm 1 \mathrm{mN} \cdot \mathrm{m}^{-1}$ measured by the pendant drop method (Krüss DSA30). The outer phase of viscosity $\eta=10^{-3} \mathrm{~Pa} \cdot \mathrm{s}$ contains fluorescein at ppm concentration and imaging is done in fluorescence in order to clearly identify the mineral oil droplet interfaces. These oil droplets, with viscosity $\eta_{\mathrm{i}}=25 \times 10^{-3} \mathrm{~Pa} \cdot \mathrm{s}$, were prepared using a microfluidic T-junction. The flow rate of both phases is set by a pressure controller (Fluigent MFCX-Flex), which enables the control of both the droplet length and positioning within order $10 \mu \mathrm{m}$. In particular, this positioning ensures that the middle of the droplet is aligned with the resistance. These non-wetting droplets, with lengths in the 
range $500 \leq L \leq 4000 \mu \mathrm{m}$, were confined in the rectangular channel (width $W=200$ or $400 \mu \mathrm{m}$, and thickness $20 \leq e \leq 60 \mu \mathrm{m}$ ) of a poly(dimethylsiloxane) microsystem (PDMS, RTV). As such, menisci and gutters have characteristic radii e/2 (Fig. 11a).

The lower surface of the microchannel is a $30 \mu \mathrm{m}$-thick PDMS layer, spin coated over a $50 \mu \mathrm{m}$-wide micropatterned resistance [20] crossing the channel at right angles. Given the geometry of the channel and the volume of the oil phase, the droplets adopt a flattened, plug-like shape. To ensure the presence of a lubricating water film at $z= \pm e / 2$, PDMS was rendered hydrophilic using oxygen plasma (FemtoScience). This treatment gives rise to thin films of water between the oil and PDMS stabilised by electrostatic forces [21]. The lubrication films are so thin, and the volume they contain is so small, that their contribution to liquid drainage will be henceforth considered negligible ; a precise justification of this assumption can be found in [22] noting that we are in the regime $1 \ll \frac{\eta_{i}}{\eta} \ll C a^{-1 / 3}$ constantly for our experiments.

In order to prepare the well-controlled, out-of-equilibrium droplet interfaces shown in Fig. 1, we first center the droplets over the heating resistance. Then the lower PDMS wall is deformed by applying a potential of $5 \mathrm{~V}$ across a $300 \Omega$ resistance. The associated Joule heating expands the lower PDMS layer. This local deformation creates a droplet confinement gradient, with the channel thickness $e$ varying along the $x$-direction as sketched in Fig. 17). Such a confinement profile is accompanied by a corresponding gradient in the out-of-plane interface curvature, which, through the Laplace law, generates a pressure gradient. In order to equalize the capillary pressure along the droplet interface, an opposing in-plane curvature is developed in the vicinity of the resistance as described by Dangla et al. [23]. We refer to this outer-phase confined region as the pocket. The droplet thus adopts a peanut-like shape (Fig. 1). In this study, we avoided large deformations leading to droplet breakup; although, in practice this, or any other, channel deformation can be used as an on-demand droplet breakup mechanism.

When the heating resistors are switched off at time $t=0 \equiv t_{\text {off }}$, the PDMS relaxes and the droplet then returns to equilibrium over a few tens of seconds. A sequence of droplet relaxation profiles is shown in Fig. 1 b). The distance between the channel side wall and the deformed interface is denoted $w(x, t)$; the maximal value of the profile at $x=0$, the pocket center, $w(0, t)=w_{0}(t)$ is called the pocket width, cf. Fig. 1. In Figs. 2a) and b) are shown a spatio-temporal evolution of the channel intensity cross-section at $x=0$ for two different 


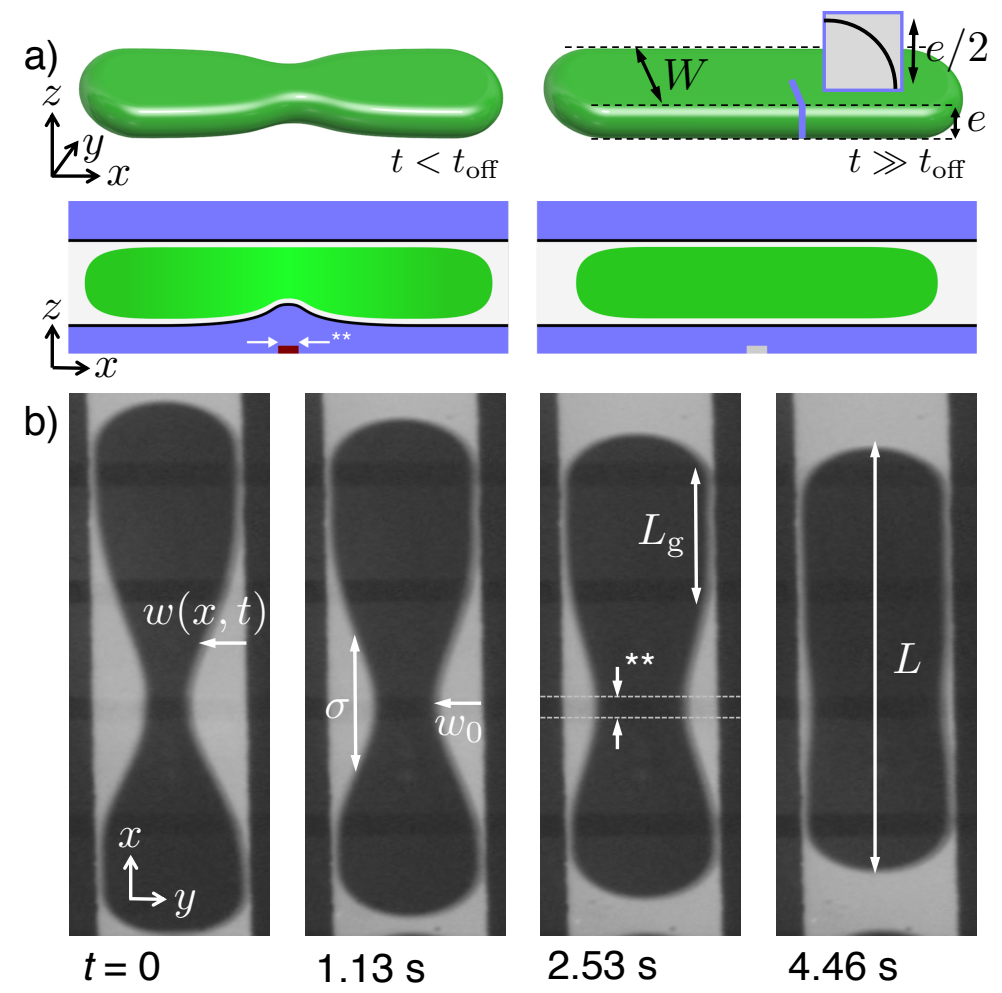

FIG. 1: a) [top] 3D sketches of: the droplet geometry shaped by the confinement in height, $e$, and width, $W$; and the fully relaxed droplet at long times. The inset schematically shows the form of the gutters, and the meniscus of radius $e / 2$. [bottom] The thermo-mechanical channel actuation leading to the droplet deformation is sketched in the $(x, z)$ plane, respectively before and soon after the voltage is extinguished at $t=0 \equiv t_{\text {off }}$ (b) Snapshots of the relaxation of the droplet. The pocket full-width half-maximum is $\sigma$, and $w(x, t)$ is the distance between the channel wall and the deformed interface, of maximal value $w_{0}(t)=w(0, t)$; we define $L_{\mathrm{g}}$ as the gutter length and $L$ as the undeformed droplet length. The location of the heating element is marked by the starred arrows.

droplet lengths. Two regimes are observed. First, the pocket relaxes abruptly over a typical time of hundreds of milliseconds. Afterwards, the relaxation proceeds much more slowly and the droplet approaches equilibrium under the constraints of the confining walls.

The first temporal regime corresponds to the thermal relaxation of the dilated PDMS. In 

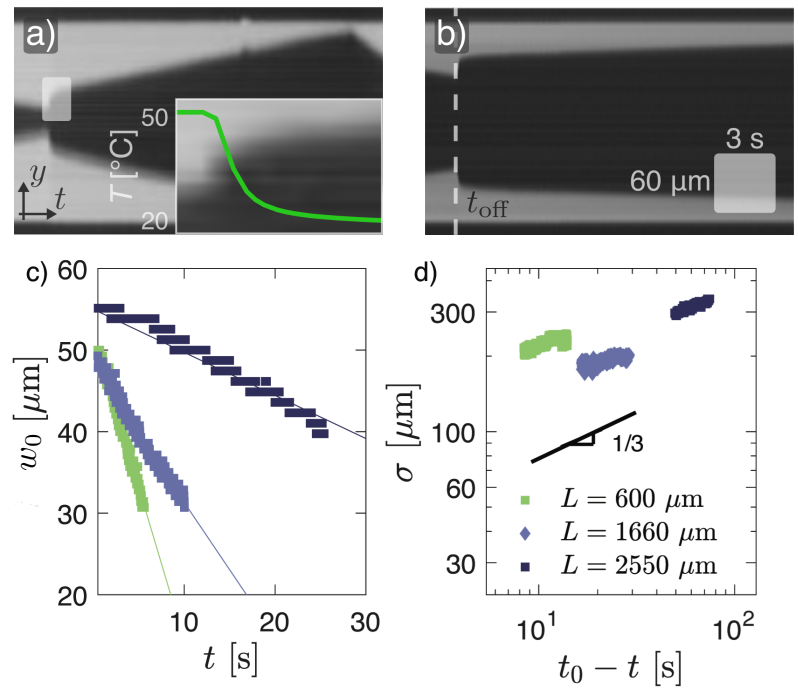

FIG. 2: Spatiotemporal evolution of the pocket for two droplets in a channel of section $e \times W=$ $30 \times 200 \mu \mathrm{m}^{2}$ with lengths a) $L=510 \mu \mathrm{m}$ and b) $L=1025 \mu \mathrm{m}$; the scale applies to both a) and b). The inset of part a) shows the experimental temperature versus time for the highlighted $1.4 \mathrm{~s}$ interval; the dashed vertical line of part b) indicates $t_{\text {off }}$. c) Plot of $w_{0}$ vs. time for three droplet lengths and d) the corresponding time dependence of the horizontal extent of the pocket, $\sigma$.

the inset of Fig. $2 \mathrm{a}$ ), the green line shows a temperature profile taken with an infrared camera (Flir Camera, SC7000), along with a zoom of the early-time relaxation; both relaxations take place over the same period of order $100 \mathrm{~ms}$, consistently with previous works reporting a similar temperature relaxation timescale [24] for similar microchannels. Over this initial period, the channel thickness $e(x)$ relaxes such that the gradient of confinement disappears and the volume available within the channel suddenly increases. As shown in the Supplementary Materials, a custom calibration method is used to quantify this newly available volume. It is therein shown to be dominantly occupied by the inner phase and is equivalent to the best measurement we can make of the channel deformation volume at $t=t_{\text {off }}$. The experimental verification of this volume conservation allows us to conclude that: i) the inner phase flows more easily than the outer phase despite the larger viscosity of the former, consistently with related experiments, theoretical and numerical works [6, 22, 25, 26]; ii) the PDMS underlayer is fully relaxed after this initial stage; and consequently iii) thermal inertia is negligible. The observation that the inner phase flows to complete the short-time relaxation suggests furthermore that the later dynamics is in turn limited by the outer phase. 
Lastly, the deformation volume of the lower channel wall is relatively small compared to the total droplet deformation and the droplet remains out of equilibrium after the fast substrate relaxation. This is how the droplet interface is set out of equilibrium in our experiment.

The second temporal regime is due to capillary-driven flow. In Figs. 2c) and d) we show the time evolution of the geometric features describing the neck: first, the pocket width $w_{0}(t)$ and second, the pocket extension, $\sigma(t)$, as shown schematically in Fig. 1 b), defined as the full-width half maximum of the pocket profile. For all our droplets, we observe that the pocket width, $w_{0}(t)$, varies linearly with time over a significant period, and extrapolates to zero thickness at a time denoted as $t_{0}$, of order ten seconds. We note also that the relaxation time increases with the droplet length for a given channel geometry as demonstrated in Fig. 22). Despite the small range of variation that is attainable with these experiments, the time evolution of the pocket extension $\sigma(t)$ is compatible with a power-law scaling $\left(t_{0}-t\right)^{n}$, with $n=1 / 3$ providing a good description of the data. In the Supplementary Materials, the robustness of this exponent is discussed in more detail.

In Fig. 3a) we show the time evolution of the full profiles in the pocket region. Based on the temporal evolutions of the pocket features found in Fig. 2 $\mathrm{b}$ ) and c), we apply the scalings describing $w_{0}(t)$ and $\sigma(t)$ to the entire profiles of Fig. 3a). Fig. 3b) depicts the rescaled curves of $w(x, t) / \tau$ as a function of $x / \tau^{1 / 3} \equiv u$, where $\tau=t_{0}-t$. Remarkably, the measured profiles for each time collapse onto a single master curve; the evolution of the water/oil interface profile is self-similar [7, 27,29]; all of our experimental observations show self-similar relaxations with same scaling exponents.

The driving force stems from the Laplace pressure gradient that drives the outer phase from the pocket to the droplet extremities through the gutters. The dynamical process is mediated by viscous dissipation in the outer phase. We showed in previous works that for interfaces driven using a temperature gradient in a 2D-confined foam, SDS interfaces display no surface tension gradient, contrary to dodecanol [30]. Therefore, even during the deformation stage, there are no Marangoni effects. Since thermal inertia is negligible, surface tension gradients are safely neglected when the heaters are turned off.

We now propose an interpretation compatible with the observed self-similar exponents, and based on confinement effects: we account for the fact that the droplet is highly confined over the cavity thickness, especially near the moving menisci where the outer phase thickness approaches just a few nanometers. Four such menisci are advancing at the pocket location, 
at the top and bottom walls, and for the two sides of the droplet [35] Due to the symmetry along the center of the channel, we only consider a single pocket. Bretherton showed that a large viscous dissipation arises in the dynamical meniscus [31, 32], which connects the flat lubricating film and the static meniscus with characteristic curvature of roughly $2 / e$ (neglecting the local in-plane curvature which is small by comparison here). The viscous drag force per unit length of the dynamical meniscus scales with the capillary number, $C a=\eta V / \gamma$ with $V$ a typical meniscus velocity. Cantat showed that $\left.F_{\text {diss }} \approx \gamma C a^{2 / 3}[32] 36\right]$. The liquid velocity in the meniscus at the top of the pocket is $V=\partial_{t} w$ and the subsequent dissipative force in the dynamical meniscus writes $F_{\text {diss }} \approx \gamma\left(\eta\left|\partial_{t} w\right| / \gamma\right)^{2 / 3}$.

The driving force is generated by the gradient of curvature along the interface, and writes in the limit of small slopes, and per unit length of the meniscus: $F_{\text {cap }} \approx \gamma e^{2} \partial_{x}^{3} w$. Written in this way, in a Brinkman approach [33], we consider that the fluid set into motion by this capillary force extends over a typical length scale $e$ away from the meniscus. By balancing
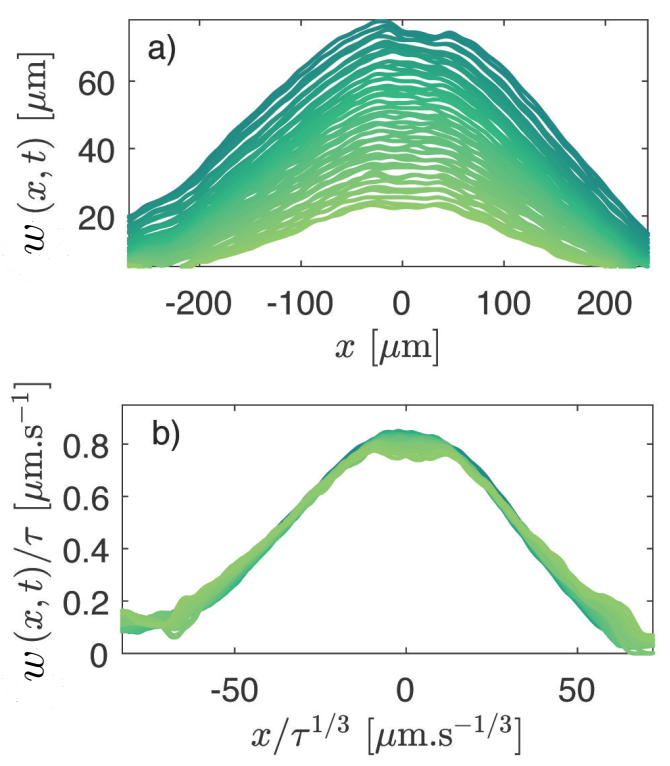

FIG. 3: a) Interface profile $w(x, t)$ in the pocket region. Blue plots illustrate the pocket profiles at early stages and green plots show the later stages; the total elapsed time is $6.9 \mathrm{~s}$ and the droplet length was $L=800 \mu \mathrm{m}$, with $e=30 \mu \mathrm{m}$ and $W=200 \mu \mathrm{m}$. b) The same profiles of the central droplet interface with $w$ rescaled by $t_{0}-t$, and $x$ by $\left(t_{0}-t\right)^{1 / 3}$. 
driving and dissipation forces:

$$
\left(\frac{\eta}{\gamma}\left|\partial_{t} w\right|\right)^{2 / 3} \sim e^{2} \partial_{x}^{3} w .
$$

Inserting the self-similar form suggested by our experiments, $w(x, t)=\tau^{\alpha} f(u)$, and requiring the resulting form of Eq. (1) to be time-invariant leads to $\alpha-9 \beta+2=0$. This relation is compatible with the observed experimental self-similar exponents.

In order to close the problem, we consider the flow from the pocket towards the droplet extremities through the gutters. The volume $\Omega(t)$ of the external phase contained in the pocket can be written $\Omega(t)=e \int w(x, t) \mathrm{d} x$. Using the self-similar expression of $w(x, t)$, $\Omega(t)=e \tau^{\alpha+\beta} \int f(u) \mathrm{d} u$. Hence $\partial_{t} \Omega=-e(\alpha+\beta) \tau^{\alpha+\beta-1} \int f(u) \mathrm{d} u$. By volume conservation, $\partial_{t} \Omega$ equals the volumetric flux $Q$ in the gutters. Assuming a Poiseuille-like flow in the gutters of cross-section of order $e^{2}, Q \approx e^{4} \Delta P\left(\eta \tilde{L}_{\mathrm{g}}\right)^{-1}$, where $\tilde{L}_{\mathrm{g}}$ is the effective gutter length defined by $\tilde{L}_{\mathrm{g}}^{-1}=\left(L_{\mathrm{g}}^{\prime}\right)^{-1}+\left(L_{\mathrm{g}}^{\prime \prime}\right)^{-1}$, cf. Fig. 4 a), assuming parallel resistances. We checked that $1 / L_{g}$ remains constant throughout the evolution within $12 \%$ for all droplets. The pressure difference is difficult to estimate precisely, but since the flow is overall driven by the bulging pocket of central curvature $-\left.\partial_{x}^{2} w\right|_{0}$, it is reasonable to assume $\Delta P \approx-\left.\gamma \partial_{x}^{2} w\right|_{0}$ [37]. Hence, $Q \approx-\left.e^{4} \gamma\left(\eta \tilde{L}_{\mathrm{g}}\right)^{-1} \partial_{x}^{2} w\right|_{0}$. Imposing volume conservation between the gutters and the pockets,

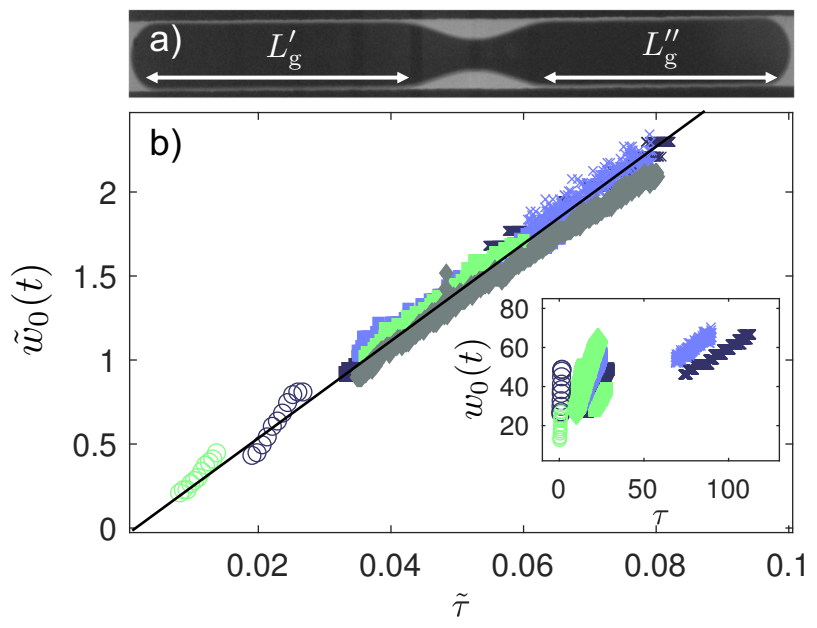

FIG. 4: a) A $1300 \mu \mathrm{m}$ long droplet in a $200 \mu \mathrm{m}$ wide channel showing the two gutter lengths used to calculate $\tilde{L}_{\mathrm{g}}$. b) Dimensionless pocket width $\tilde{w}_{0}=w_{0} / e$ as a function of dimensionless time $\tilde{\tau}=\tau \tau_{\mathrm{d}}^{-1}$ for different sets of experiments: for $\{*, *, \mathbf{\square}, \mathbf{\square}, \mathbf{\square}, \bullet, \bullet, \diamond\}$ we have $[L, e, W]=\{[1150,20,400],[950,20,400],[800,20,400],[1025,30,200],[750,20,200],[540,20,200]$, $[350,20,200],[804,59,200]\}$ all in $\mu \mathrm{m}$. 
$-\partial_{t} \Omega=Q$, and the observed self-similarity on $Q$ then leads to

$$
e(\alpha+\beta) \tau^{\alpha+\beta-1} \int f(u) \mathrm{d} u \approx-\frac{\gamma \tau^{\alpha-2 \beta} f^{\prime \prime}(0)}{\eta \tilde{L}_{\mathrm{g}}} e^{4} .
$$

In Equation (2) all the terms, except the ones with $\tau$, are time independent giving $\alpha-2 \beta=$ $\alpha+\beta-1$. By coupling the self-similar form of Equation (1) and Equation (2), we find: $\alpha=1, \beta=1 / 3$. These model predictions agree quantitatively with our experimental observations, demonstrating the dominating effect of the menisci and gutters located at the confining walls on the drainage dynamics, which emphasizes the 3D nature of the flows considered here.

Finally, it is possible to define a characteristic drainage time to rescale all of the experimental pocket widths $w_{0}(t)$. Mass conservation consideration gives a relation between the velocity of the meniscus at the center of the pocket and the flux in the gutters $w \partial_{t} w \approx Q / e$. As a result, the mean velocity of the pocket meniscus can be written $\frac{w}{e} \frac{\Delta w}{\tau_{\mathrm{d}}}=-\gamma\left(\eta \tilde{L}_{\mathrm{g}}\right)^{-1} e^{2} \partial_{x}^{2} w$, where the time $\tau_{\mathrm{d}}$ is the typical drainage time during which the pocket width reaches the channel width $\Delta w \approx W$ with constant velocity. Fig. 1 suggests that $\partial_{x}^{2} w$ is of the order of $W^{-1}$; this is shown more quantitatively in the Supplementary Material. We therefore define $\tau_{\mathrm{d}}=W \eta \tilde{L}_{\mathrm{g}} / \gamma e$. The time evolution of the pocket width $w_{0}(\tau)$ can thus be represented using the dimensionless variables $\tilde{w}$ and $\tilde{\tau}$ with $\tau=\tau_{\mathrm{d}} \tilde{\tau}$ and $w=e \tilde{w}$. In Fig. 4, all the rescaled data remarkably collapse onto a single linear curve, whatever the droplet length, channel width or cavity thickness. As such, momentum conservation in the advancing pocket meniscus coupled to a volume conservation in the gutters captures faithfully the experimental observations of the relaxation of the droplet for a wide range of length, channel height and width.

To conclude, we have predicted the relaxation dynamics of an intrinsically threedimensional flow with a scaling analysis considering capillary driving forces and viscous dissipation. Critically, this dissipation is dominated by that in the gutters and in the menisci. Such confined relaxation, ubiquitous in real systems, allowed us to go beyond symmetry-based models on interface relaxation. In the context of studying confined multiphase flows [13, 14], we believe this approach opens the way to study the effect of topographic inhomogeneities on the deformation and relaxation of drops or bubbles.

This work was supported by CNRS, ESPCI Paris, Agence Nationale de la Recherche (ANR) under the grant 13-BS09-0011-01, IPGG (Equipex ANR-10-EQPX-34 and Labex 
ANR-10-LABX-31), PSL (Idex ANR-10-IDEX-0001-02), and Dim-NanoK Région Ile de France.

[1] J. M. Rallison, Annu. Rev. Fluid Mech. 16, 45 (1984).

[2] H. A. Stone and L. G. Leal, J. Fluid Mech. 206, 223 (1989).

[3] D. Y. C. Chan, E. Klaseboer, and R. Manica, Soft Matter 7, 2235 (2011).

[4] J. de Ruiter, R. Lagraauw, D. van den Ende, and F. Mugele, Nature Phys. 11, 48 (2014).

[5] C. Josserand and S. T. Thoroddsen, Annu. Rev. Fluid Mech. 48, 365 (2016).

[6] L. Bluteau, M. Bourrel, N. Passade-Boupat, L. Talini, E. Verneuil, and F. Lequeux, Soft Matter 13, 1384 (2017).

[7] J. D. McGraw, T. Salez, O. Bäumchen, E. Raphaël, and K. Dalnoki-Veress, Phys. Rev. Lett. 109, $128303(2012)$.

[8] T. Squires and S. Quake, Rev. Mod. Phys. 77, 977 (2005).

[9] N. Bremond, A. R. Thiam, and J. Bibette, Phys. Rev. Lett. 100, 024510 (2008).

[10] T. Cubaud, Phys. Rev. E 80, 026307 (2009).

[11] C. N. Baroud, F. Gallaire, and R. Dangla, Lab Chip 10, 2032 (2010).

[12] P. T. Brun, M. Nagel, and F. Gallaire, Phys. Rev. E 88, 043009 (2013).

[13] C. Odier, B. Levaché, E. Santanach-Carreras, and D. Bartolo, Physical review letters 119, 208005 (2017).

[14] V. Hourtane, H. Bodiguel, and A. Colin, Physical Review E 93, 032607 (2016).

[15] D. R. Link, S. L. Anna, D. A. Weitz, and H. A. Stone, Phys. Rev. Lett. 92, 054503 (2004).

[16] H. A. Stone, Annu. Rev. Fluid Mech. p. 26 (1994).

[17] Q. Brosseau, J. Vrignon, and J. C. Baret, Soft Matter 10, 3066 (2014).

[18] L. Zhu and F. Gallaire, J. Fluid Mech. 798, 955 (2016).

[19] P. Tabeling, Introduction to Microfluidics (Oxford University Press, 2005).

[20] V. Miralles, A. Huerre, H. Williams, B. Fournie, and M. C. Jullien, Lab Chip 15, 2133 (2015).

[21] A. Huerre, M.-P. Valignat, A. C. Maggs, O. Theodoly, and M.-C. Jullien, Appl. Phys. Lett. 111, 221601 (2017).

[22] S. R. Hodges, O. E. Jensen, and J. M. Rallison, J. Fluid Mech. 501, 279 (2004).

[23] R. Dangla, C. Kayi, and C. Baroud, Proc. Natl. Acad. Sci. 110, 853 (2012). 
[24] B. Selva, P. Mary, and M.-C. Jullien, Microfluidics and Nanofluidics 8, 755 (2010).

[25] C.-W. Park and G. Homsy, Journal of Fluid Mechanics 139, 291 (1984).

[26] L. Keiser, K. Jaafar, J. Bico, and É. Reyssat, Journal of Fluid Mechanics 845, 245 (2018).

[27] G. Barenblatt, Scaling (Cambridge University Press, 2003).

[28] M. C. Dallaston, D. Tseluiko, Z. Zheng, M. A. Fontelos, and S. Kalliadasis, Nonlinearity 30, $2647(2017)$.

[29] J. Eggers and M. Fontelos, Nonlinearity 22, R1 (2009).

[30] V. Miralles, E. Rio, I. Cantat, and M.-C. Jullien, Soft Matter 12, 7056 (2016).

[31] F. P. Bretherton, J. Fluid Mech. 10, 166 (1961).

[32] I. Cantat, Phys. Fluids 25, 031303 (2013).

[33] P.-T. Brun, M. Nagel, and F. Gallaire, Physical Review E 88, 043009 (2013).

[34] D. A. Hoang, L. M. Portela, C. R. Kleijn, M. T. Kreutzer, and V. van Steijn, J. Fluid Mech. 717, R4 (2013).

[35] We note that four menisci are also receding at the droplet extremities (top and bottom walls at the two extremities). As the scalings are similar for advancing and receding meniscus, our scaling analyses to follow are unchanged.

[36] More precisely, the meniscus dissipation is moderated by the projection of the fluid velocity normal to the meniscus. In this case we write the viscous drag per unit length of the dynamical meniscus is $F_{\text {diss }} \approx \gamma(\cos \theta)^{2 / 3} \mathrm{Ca}^{2 / 3}$. The dissipation as written in the text considers the main portion of the dissipation only which is at the top of the pocket where the fluid velocity is normal to the meniscus.

[37] We omit details concerning the matching zone between the pocket and the gutters. However, Hoang et al. have shown that, provided the curvature of the pocket is smaller than the one at the entrance of the gutters, the flow is globally driven by the central curvature [34]. The driving pressure gradient for this flow is therefore approximated using the central curvature of the pocket which is always bigger than the one at the matching zone in our experiments. 